\title{
Anticancer Activity of Thymoquinone Cubic Phase Nanoparticles Against Human Breast Cancer: Formulation, Cytotoxicity and Subcellular Localization
}

\author{
Mohammed M Mehanna (iD) ${ }^{1,2, *}$ \\ Rana Sarieddine ${ }^{3, *}$ \\ Jana K Alwattar ${ }^{2}$ \\ Racha Chouaib ${ }^{3}$ \\ Hala Gali-Muhtasib (iD ${ }^{3}$ \\ 'Department of Industrial Pharmacy, \\ Faculty of Pharmacy, Alexandria \\ University, Alexandria, Egypt; \\ ${ }^{2}$ Department of Pharmaceutical \\ Technology, Faculty of Pharmacy, Beirut \\ Arab University, Beirut, Lebanon; \\ ${ }^{3}$ Department of Biology, Faculty of Arts \\ and Sciences, American University of \\ Beirut, Beirut, Lebanon
}

*These authors contributed equally to this work

This article was published in the following Dove Press journal:

International Journal of Nanomedicine

Introduction: Triple negative breast cancer is an aggressive disorder which accounts for at least $15 \%$ of breast cancer diagnosis and a high percentage of breast cancer morbidity, hence intensive research efforts are focused on the development of effective therapies to overcome the disease. Thymoquinone (TQ), the bioactive constituent of Nigella sativa, exhibits anticancer activity, yet its translation to the clinic is hindered by its poor bioavailability and lack of quantification method in blood and tissues. To overcome these limitations, cubosomes were utilized for the encapsulation and delivery of this anticancer molecule.

Methods: Thymoquinone loaded cubosomes were prepared through the emulsification homogenization method. The physicochemical characteristics, including particle size, zeta potential, morphology and entrapment efficiency, were studied. Moreover, the in vitro antitumor activity was tested on breast cancer cell lines (MCF-7 and MDA-MB-231) and compared to non-tumorigenic cell line (MCF-10A). Subcellular localization, cellular uptake and apoptotic effects of the formulations were assessed.

Results: The results revealed that the TQ loaded cubosomal formulation exhibited a mean particle size of $98.0 \pm 4.10 \mathrm{~nm}$ with narrow unimodal distribution. The high entrapment efficiency $(96.60 \pm 3.58 \%)$ and zeta potential $(31.50 \pm 4.20 \mathrm{mV})$ conceived the effectiveness of this nanosystem for TQ encapsulation. Cell viability in both breast cancer cell lines demonstrated a dose-dependent decrease in response to treatment with free TQ or TQ-loaded cubosomes, with enhanced antitumor activity upon treating with the latter formulation. A significant increase in apoptotic bodies and cleaved caspase 3 was observed upon treatment of MDA-MB-231 cells with either TQ or TQ-loaded cubosomes. Localization and trafficking studies unveiled that cubosomes accumulate in the cytoplasm of the studied breast cancer cell lines.

Discussion: Our results show that thymoquinone encapsulation in cubosomal nanoparticles provides a promising anticancer drug delivery system with the ability to label, detect and subsequently trace it within the human cells.

Keywords: antitumor, breast cancer, cubosomes, endocytosis, nanoparticles, thymoquinone, uptake

(t) Department of Biology, Faculty of Arts and Sciences, American University of Beirut, Riad El Solh, Beirut II 07 2020,

Lebanon

Email amro@aub.edu.lb

Jana K Alwattar

Department of Pharmaceutical

Technology, Faculty of Pharmacy, Beirut

Arab University, Riad El Solh, Beirut I 107

2020, Lebanon

Email janawattar@gmail.com

\section{Introduction}

Cancer is one of the most distressing diseases and the second leading cause of death with 18.1 million new cases and 9.6 million deaths reported in 2018 with no definite cure yet. ${ }^{1}$ Breast cancer is the most frequent type of cancer in women, representing $30 \%$ of the newly diagnosed cancer cases, with a mortality estimate of 
about $15 \%$ of deaths in $2019 .^{2}$ Depending on the breast cancer type and its severity, conventional treatment modalities include chemotherapy, radiotherapy, surgery, hormonal and targeted therapies. However, these strategies lead to several serious side effects due to their nonselectivity, high-dose requirement, poor bioavailability of drugs and systemic toxicity. ${ }^{3}$ Therefore, researchers are looking for alternative chemical compounds with potential anticancer activity that are less toxic such as the naturally extracted compounds resveratrol, ${ }^{4}$ purpurin, ${ }^{5}$ camptothecin, ${ }^{6}$ curcumin, ${ }^{7}$ paclitaxel ${ }^{8}$ and various other plant derived compounds.

Nigella sativa, commonly known as the black seed, has been thoroughly studied over the years, exhibiting several pharmacological actions through its main active constituent thymoquinone (TQ). Immune system stimulatory, antiinflammatory, hypotensive, hepatoprotective, anticancer and hypoglycemic properties of TQ were previously studied. ${ }^{9}$ TQ was able to modulate nine of the ten hallmarks of cancer. ${ }^{9}$ Besides its promising anticancer effects against several solid tumors including breast cancer, TQ is inexpensive, readily available from plants and non-toxic to normal cells and tissues. ${ }^{10}$ However, TQ has not been assessed in the clinical setting against human cancers due to its hydrophobic nature, poor solubility and bioavailability, in addition to its strong tendency to be plasma protein bounded, therefore, hindering it from reaching the tumor site. ${ }^{11,12}$

Encapsulation of TQ into nanocarriers based systems is being extensively studied and has so far shown encouraging results especially in terms of enhancing TQ bioavailability, biodistribution, cellular delivery and targeting of cancer cells. ${ }^{13-15}$ Anticancer activity of nanosystems is directly affected by the particle size of the system and accordingly the nanoparticle (NP) size recommended by the National Cancer Institute for neoplastic cell targeting is less than $100 \mathrm{~nm} .{ }^{16}$ The nanocarriers utilized can carry more than one TQ molecule, thus increasing the concentration of the drug delivered to the tumor site without affecting the non-cancerous tissue. Since highly aggressive tumors form fenestrated vasculature due to their deregulated and leaky nature and the poor lymphatic drainage at the tumor sites, nanoparticle-encapsulated drugs could be retained and accumulated within the cancerous mass, thus maximizing drug contact with decreasing drug exposure to normal tissues and reducing side effects. This enhanced permeability and retention effect (EPR) results in passive drug targeting to the tumors, which confers an advantage for NP encapsulated drugs over their free forms. ${ }^{17,18}$ During the past decade nanocarrier systems have been investigated to increase the therapeutic index of drugs at the target site. The advances of these vectors in cancer therapy include liposomes, cyclodextrin based nanoparticles, polymeric micelles, nanospheres and cubosomes, among many other carriers. ${ }^{19-23}$

Self-assembled lipid liquid-crystalline nanostructures, known as cubosomes, are promising drug delivery systems due to their unique feature of possessing a large hydrophobic volume which allows efficient encapsulation of poor water-soluble drugs in appropriate concentrations. $^{24,25}$ The formation of the bi-continuous cubic phases relies on the combination of lipids and stabilizers. The most utilized system to construct the cubosomal nanoparticles is monooleins, which spontaneously forms a cubic phase upon its incorporation into an aqueous medium. Monooliens swell in water thus producing several characteristic lyotropic liquid crystalline structures. In addition, they are biodegradable, biocompatible and are considered as relatively safe which makes them favorable compounds for cubosomal formation. ${ }^{26}$ The second major component within the cubic phase structures is the stabilizer. These materials may include polyvinyl alcohol, Tween ${ }^{\circledR} 80$, Laponite ${ }^{\mathbb{R}}$ XLG, polyethylene oxide (PEO), although the most frequently studied molecules are the amphiphilic nonionic triblock copolymer Poloxamer ${ }^{\circledR}$ F-127 which consists of hydrophobic polyoxypropylene and hydrophilic PEO blocks. The hydrophobic fraction is absorbed onto or is incorporated at the surface of the stabilized particle while the hydrophilic blocks extend over the surface of the particle, thus providing a steric shielding and further stabilization of the nanoparticles. ${ }^{27}$ Most importantly, cubosomes are $\mathrm{pH}$-responsive nanocarriers that do not release their loaded drugs except in the acidic environment which is the case at the tumor site, thus increasing selectivity of the drug and reducing systemic toxicities. ${ }^{28}$

In this study, we focused on the preparation of a novel TQ-loaded cubosomal formulation as a potential system to enhance the antitumorigenic activity of thymoquinone. The formulation physicochemical features viz., particle size, zeta potential, morphology, and entrapment efficiency were investigated. The in vitro antitumor activity of TQloaded cubosomal formulation was examined on breast cancer (MCF-7 and MDA-MB-231) and non-tumorigenic breast (MCF-10A) cell lines. Furthermore, cellular uptake 
and subcellular localization were evaluated via Nile red labeled TQ-loaded cubosomes.

\section{Materials and Methods}

\section{Chemicals and Media}

Thymoquinone, was obtained from Sigma Aldrich ( $\mathrm{St}$ Louis, Missouri, USA). Peceol ${ }^{\circledR}$ (glyceryl monooleate, GMO), was a generous gift from Gattefosse (Lyon, France). Poloxamer ${ }^{\circledR} 407$ (PLX), Nile Red (NR), trypsin-EDTA, DMSO, MTT (3-(4,5-Dimethylthiazol-2-yl)2,5-diphenyltetrazolium bromide), trypan blue, $\operatorname{Triton}^{\mathbb{B}}$ X100 and methanol were purchased from Sigma Aldrich (St Louis, Missouri, USA). DAPI stain was purchased from Abcam (Cambridge, UK).

MCF-10A, non-tumorigenic breast cell line, MCF-7, breast cancer cells and MDA-MB-231, aggressive breast cancer cells, were procured from American Type Culture Collection (ATCC), USA. Dulbecco's phosphate-buffered saline (PBS), horse serum, fetal bovine serum (FBS), penicillin-streptomycin (P/S), epidermal growth factor, hydrocortisone, insulin and cholera toxin were purchased from Sigma Aldrich (St Louis, Missouri, USA). Dulbecco's Modified Eagle Medium (DMEM) and DMEM-F12 cell culture media were purchased from Lonza (Verviers, Belgium). Caveolin 1N-20 rabbit polyclonal, transferrin H-65 rabbit polyclonal, EEA-1 E-8 mouse monoclonal IgG and lamp-1 H4A3 mouse monoclonal $\operatorname{IgG}$ antibodies were purchased from Santa Cruz Biotechnology (Paso Robles, California, USA). Cleaved caspase 3 monoclonal antibody was purchased from Cell Signaling Technology (Danvers, Massachusetts, USA). Anti-mouse secondary antibody alexa 488 and anti-rabbit secondary antibody alexa 568 were purchased from Invitrogen (Carlsbad, California, USA).

\section{Preparation of Self-Assembled Cubic Liquid Crystalline Nanoparticles (LCNPs)}

In order to prepare the cubosomes, emulsification method was adopted utilizing glyceryl monooleate (GMO) as the lipid phase in the presence of pluronic F127 (PLX) as the stabilizer as previously described by Elgindy et al. ${ }^{29}$ Briefly, a calculated amount of lipidic phase was mixed with PLX and heated using thermostatically controlled water bath (FALC, WB-MF24, Treviglio (BG)-Italy) at $70 \pm 2{ }^{\circ} \mathrm{C}$. To the melted lipid phase mixture, thymoquinone $(100 \mathrm{mg} / \mathrm{mL})$ was added and allowed to stand for 2 minutes in an ultrasonic bath to ensure complete solubilization. The formed dispersed system was injected using a syringe into a preheated aqueous phase containing PLX. The mixture was emulsified using a high-speed rotor-stator homogenizer set at 22,000 rpm for 3 cycles of 10 minutes each. The final composition of the cubosomes was 4.5/0.75/94.75 (wt\%) GMO/PLX/water and 0.44/4.5/0.75/94.31 (wt\%) TQ/GMO/PLX/water for the TQ doped cubosomes. The samples were maintained in light protected vials at ambient temperature for further investigations.

\section{Preparation of Labeled LCNPS}

Florescent cubosomes were labeled using Nile red (NR), a lipid soluble dye, which was selected as it presented nonsignificant alteration to the particle size, zeta potential and drug loading of the cubosomal preparation. A stock solution of fluorescent GMO was prepared by dissolving NR with a final concentration $0.5 \mathrm{mg} / \mathrm{g}$ into GMO and left to equilibrate for 48 hours under mild stirring at room temperature. ${ }^{30}$ The florescent-loaded oil was then utilized for the preparation of the self-assembled cubosomes and thymoquinone loaded cubosomes as previously mentioned.

\section{Particle Size and Polydispersity Index (PDI) Determination}

The particle size and size distribution of prepared thymoquinone loaded self-assembled cubosomes were determined using the dynamic light scattering technique employing Zeta sizer 2000 (Malvern instruments, UK). For optimal sensitivity, thymoquinone loaded cubosomes were diluted with Milli Q water and sonicated for 5 minutes prior to measurement. All measurements were performed in triplicate at $25 \pm 0.5{ }^{\circ} \mathrm{C} .{ }^{31}$

\section{Zeta Potential Measurement}

To confirm the physical stability of the prepared TQ-loaded self-assembled cubosomal dispersion, zeta potential (ZP) was evaluated utilizing Zeta sizer 2000 (Malvern instruments, UK), where values were determined through the dispersion technology software. Prior to measurement of each dispersion, sample dilution using Milli Q water was carried out. All determinations were performed in triplicate. ${ }^{31}$

\section{Morphological Evaluation of Thymoquinone Loaded LCNPS}

The morphological structure of thymoquinone-loaded cubosomes was examined at $80 \mathrm{kV}$ using transmission 
electron microscopy (TEM) (JEM-100 CX, JEOL, Japan). The dispersion was diluted with water and sonicated, then a drop of the dispersion was added to a copper-coated grid forming a thin film. Prior to examination, the samples were stained with phosphotungstic acid solution $(2 \% \mathrm{w} / \mathrm{v}$, pH 6.8) and air dried.

\section{Entrapment Efficiency of Thymoquinone in LCNPS}

To quantify the thymoquinone content entrapped in cubosomes, free unencapsulated thymoquinone was separated from the cubosomal formulation through transferring $0.5 \mathrm{~mL}$ of the loaded dispersion into a Vivaspin ${ }^{\circledR}$ ultrafilter centrifuge tube with a Mwt cut 10,000 (Sartorius, Germany). The dispersion was centrifuged at $5000 \mathrm{rpm}$ (Sigma 3-30KS centrifugation, Germany) for 15 minutes at $4 \pm 0.5{ }^{\circ} \mathrm{C}$. The amount of thymoquinone in the recovery chamber was quantified by high pressure liquid chromatography (HPLC). The entrapment efficiency of thymoquinone was calculated as follows: ${ }^{32}$

$$
\begin{aligned}
\% \mathrm{EE}= & (\text { Total weight of } T Q \text { added } \\
& - \text { Weight of unentrapped } T Q) / \text { Total weight } \\
& \text { of } T Q) \times 100
\end{aligned}
$$

\section{Analytical System for Thymoquinone Determination}

The analysis was performed through a validated reverse HPLC method employed for quantifying thymoquinone content. $^{33}$ Chromatographic analysis was performed using an Agilent technology (Waldbronn, Germany) equipped with BDS HYPERSIL C18 column (250x $4.6 \mathrm{~mm} \mathrm{LD}$ ), autosampler, quaternary pump and photo diode array detector. An isocratic mobile phase of water: acetonitrile (45:55\%) mixture was eluted at a flow rate of $1 \mathrm{~mL} / \mathrm{min}$ at $25 \pm 0.5{ }^{\circ} \mathrm{C}$ and the column effluent was detected at $254 \mathrm{~nm}$ wavelength with a total run time of 15 minutes. Under these conditions, thymoquinone retention time was approximately 7.42 minutes. Thymoquinone concentration was computed via calibration curve of plotted peak area versus concentration. ${ }^{33}$

\section{Ex vivo Hemolysis Assay}

For hemolysis assay of TQ loaded cubosomal formulation and free cubosomal formulation ${ }^{30,34}$,fresh human blood samples stabilized by ethylenediaminetetraacetic acid, were centrifuged at $4 \pm 2{ }^{\circ} \mathrm{C}, 2000 \mathrm{rpm}$ for 10 minutes to separate the red blood cells (RBCs). The precipitated RBCs were washed with PBS ( $\mathrm{pH}=7.4)$. RBCs suspension was then diluted with PBS and $50 \mu \mathrm{L}$ of RBCs were added to an increasing volume of cubosomal formulation with a final volume of $250 \mu \mathrm{L}$. The positive control was treated with Triton ${ }^{\circledR}$ X100 while the negative control was established by adding PBS. The tested prepared samples were shaken at $37 \pm 2{ }^{\circ} \mathrm{C}$ for 2 hours, followed by centrifugation at $8000 \mathrm{rpm}$ for 2 minutes, the hemoglobin released in the supernatant was determined using UVvisible spectrophotometer at $540 \mathrm{~nm}$. Percentage hemolysis was calculated according to the following formula:

$$
\begin{gathered}
\text { Hemolysis }(\%)=\left(\text { Absorption }_{\text {sample } \left.- \text { Absorption }_{\text {control- }}\right)}\right) \\
/\left(\text { Absorption }_{\text {control }+}-\text { Absorption }_{\text {control- }-}\right) \times 100
\end{gathered}
$$

\section{In vitro Anticancer Studies \\ Cell Culture and Treatment}

MCF-10A, non-tumorigenic breast cells, were grown in DMEM/F-12 cell culture media supplemented with $1 \%$ penicillin/streptomycin ( $\mathrm{P} / \mathrm{S}$ with penicillin at 10,000 units and streptomycin at $10 \mathrm{mg} / \mathrm{mL}$ ), $5 \%$ horse serum, $20 \mathrm{ng} / \mathrm{mL}$ epidermal growth factor, $0.5 \mathrm{mg} / \mathrm{mL}$ hydrocortisone, $100 \mathrm{ng} / \mathrm{mL}$ cholera toxin, and $10 \mu \mathrm{g} / \mathrm{mL}$ insulin. MDA-MB-231, aggressive breast cancer cells, were grown in DMEM cell culture media supplemented with $10 \%$ heat-inactivated FBS and 1\% penicillin and streptomycin. MCF-7, breast cancer cells, were grown in DMEM cell culture media supplemented with $10 \%$ heat-inactivated FBS, $1 \% \mathrm{P} / \mathrm{S}, 5 \%$ sodium pyruvate and $5 \%$ non-essential amino acids. All cells were maintained in a humidified atmosphere of $5 \%$ carbon dioxide at $37 \pm 2{ }^{\circ} \mathrm{C}$.

\section{Cell Viability Assay}

Viable cells can reduce the yellow tetrazole MTT (3-(4,5-Dimethylthiazol-2-yl)-2,5-diphenyltetrazolium

bromide) dye into a purple insoluble formazan product, the absorbance of which was recorded at $595 \mathrm{~nm}$. MTT was used to determine the effects of TQ, TQ-loaded cubosomes, and blank cubosomes on the viability of MCF-7 and MDA-MB-231 breast cancer cells and on the nontumorigenic MCF-10A breast cell line. Cells were seeded in 96-well plates at a density of 10,000 cells well. Thymoquinone was dissolved in methanol and diluted with the cell culture media with final methanol concentration of $0.1 \%$. Both TQ loaded and blank cubosomes were diluted with cell culture media. All treatments were 
performed at 50\% confluency. After 24 hours, the medium was removed, and the cells were incubated for 3 hours with MTT solution ( $1 \mathrm{mg} / \mathrm{mL}$ in PBS). After 3 hours, the solution containing MTT dye was removed and replaced by isopropanol to dissolve the formazan crystals, the formed solution absorbance at $595 \mathrm{~nm}$ using a microplate reader was measured. Cellular viability was expressed as a percentage of cell viability of treated cells relative to untreated controls. All experiments were performed at least three times.

\section{Active Caspase 3 Detection}

MDA-MB-231 cells were plated on cover slips in 12 well plates at a density of 60,000 cells $/ \mathrm{mL}$. After overnight incubation, the medium was removed and the cells were treated for 24 hours with either TQ-loaded cubosomes, blank cubosomes, or NR-labeled TQ-loaded cubosomes. After treatment, the cells were washed twice with PBS and fixed at room temperature for 20 minutes with $4 \%$ formaldehyde, which was then removed. The cells were washed three times in PBS before permeabilization with $0.2 \%$ Triton ${ }^{\circledR}$ solution for 10 minutes. After two successive 5 minute washes in PBS, the slides were blocked in $2 \%$ bovine serum albumin (BSA) for 2 hours. Activated caspase 3 antibody was subsequently diluted (1:500) in 1\% BSA and incubated separately with the cells overnight at $4{ }^{\circ} \mathrm{C}$. The next day, the primary antibodies were removed, and the cells were washed twice before incubation for 1 hour with rabbit secondary antibodies diluted (1:200) in $0.2 \%$ BSA. The secondary antibody was then removed, and the cells were washed twice in PBS before the nuclei DAPI staining and mounting on a glass slide. Imaging and visualization were performed using the microscope Zeiss Axio (Zeiss, Germany).

\section{Cellular Uptake of LCNPS Formulations}

Uptake of fluorescent TQ-loaded cubosomes was determined qualitatively by the fluorescent microscope Zeiss Axio (Zeiss, Germany). For microscopical analysis, 70,000 cells $/ \mathrm{mL}$ of MCF-7 cells and 60,000 cells $/ \mathrm{mL}$ of MDA-MB-231 cells were plated on cover slips in 12-well plates, in $1 \mathrm{~mL}$ of respective growth medium. After treatment with NR-labeled TQ-loaded cubosomes at the indicated time points and concentrations, the cells were rinsed twice with PBS, and fixed with $4 \%$ formaldehyde solution at room temperature for 20 minutes. DAPI was used to stain the nuclei before mounting on a glass slide. Imaging and visualization were performed using the microscope Zeiss Axio (Zeiss, Germany).

\section{Subcellular Localization of Liquid Crystalline Nanoparticles}

MCF-7 cells (70,000 cells $/ \mathrm{mL})$ and MDA-MB-231 cells $(60,000$ cells) were plated on cover slips in 12 well plates. After overnight incubation in $1 \mathrm{~mL}$ of respective growth medium, the medium was removed and the cells were subjected for 30 minutes to either TQ, blank NR-labeledcubosomes, or NR-labeled TQ-loaded cubosomes. After treatment, the cells were washed twice with PBS and fixed at room temperature for 20 minutes with $4 \%$ formaldehyde which was then removed, and the cells were washed three times with PBS before permeabilization in $0.2 \%$ Triton $^{\circledR}$ solution for $10 \mathrm{~min}$ utes. After two successive 5 minute washes with PBS, the cells were blocked in 2\% BSA for 2 hours. Caveolin, transferrin, lamp-1 and EEA-1 antibodies were subsequently diluted (1:100) with $1 \%$ BSA and incubated separately with the cells overnight at $4 \pm 0.5^{\circ} \mathrm{C}$. After 24 hours, the primary antibodies were removed, and the cells were washed twice before incubation for 1 hour with mouse or rabbit secondary antibodies diluted $(1: 200)$ with $0.2 \%$ BSA. The secondary antibody was then removed and the cells were washed twice with PBS before staining the nuclei with DAPI and mounting on a glass slide. Imaging and visualization were performed using the microscope Zeiss Axio (Zeiss, Germany).

\section{Statistical Analysis}

The experiments were independently performed at least in triplicate. Data are presented as mean \pm standard error (SEM). Statistical analysis was performed by ANOVA with significance set at $\mathrm{p}$ value 0.05 .

\section{Storage Stability Evaluation}

Stability of thymoquinone-loaded cubosomes over three months were evaluated through physicochemical characteristics. The freshly prepared TQ cubosomal formulations were placed in glass vials and kept at room temperature for three months $\left(25 \pm 2{ }^{\circ} \mathrm{C}\right)$. Evaluation of physical stability of the formulations was determined through examining their particle size, PDI and zeta potential where samples were withdrawn at definite time intervals.

\section{Results \\ Characterization of LCNPs and Thymoquinone Loaded LCNPs}

Various physicochemical characteristics of the developed blank cubosomes and thymoquinone loaded cubosomal preparations are shown in Table $1(\mathrm{n}=3)$. The size for 
Table I Physicochemical Characteristics of Blank Cubosomes and Thymoquinone Loaded Cubosomal Formulation

\begin{tabular}{|l|l|l|}
\hline & $\begin{array}{l}\text { Blank } \\
\text { Cubosomes }\end{array}$ & $\begin{array}{l}\text { Thymoquinone Loaded } \\
\text { Cubosomes }\end{array}$ \\
\hline Particle size & $96.40 \pm 2.5 \mathrm{Im}$ & $98.00 \pm 4.10 \mathrm{~nm}$ \\
PDI & 0.180 & 0.234 \\
Zeta potential & $-31.50 \pm 4.2 \mathrm{mV}$ & $-34.70 \pm 2.80 \mathrm{mV}$ \\
$\begin{array}{l}\text { Entrapment } \\
\text { efficiency }\end{array}$ & & $96.60 \pm 3.58 \%$. \\
\hline
\end{tabular}

the blank cubosomes was lower than that of the TQ loaded formulation with a particle size equal to $96.40 \pm 2.51 \mathrm{~nm}$ while that of the formulated thymoquinone loaded cubosomes was $98 \pm 4.10 \mathrm{~nm}$. Moreover, the polydispersity index was lower than 1.0 in both systems with 0.18 and 0.234 for blank cubosomes and TQ-loaded cubosomal formulation, respectively. The measured zeta potentials of the various cubosomal preparations were of -34.70 $\mathrm{mV}$ and $-31.50 \mathrm{mV}$ as shown in Table 1 . The morphological structure was studied through transmission electron microscopy and revealed a clear cubic structure of TQloaded formulation as shown in Figure 1. The amount of drug encapsulated in the cubic phases in relation to the total amount of drug consumed during the preparation revealed an entrapment efficiency of $96.60 \%$.

The hemocompatibility analysis has been recognized to be essential for the evaluation of biosafety of the nanocarrier system on erythrocytes during parenteral administration. Hence, to evaluate the safety of the TQ-loaded cubosomal formulation, hemolytic activity of a series of

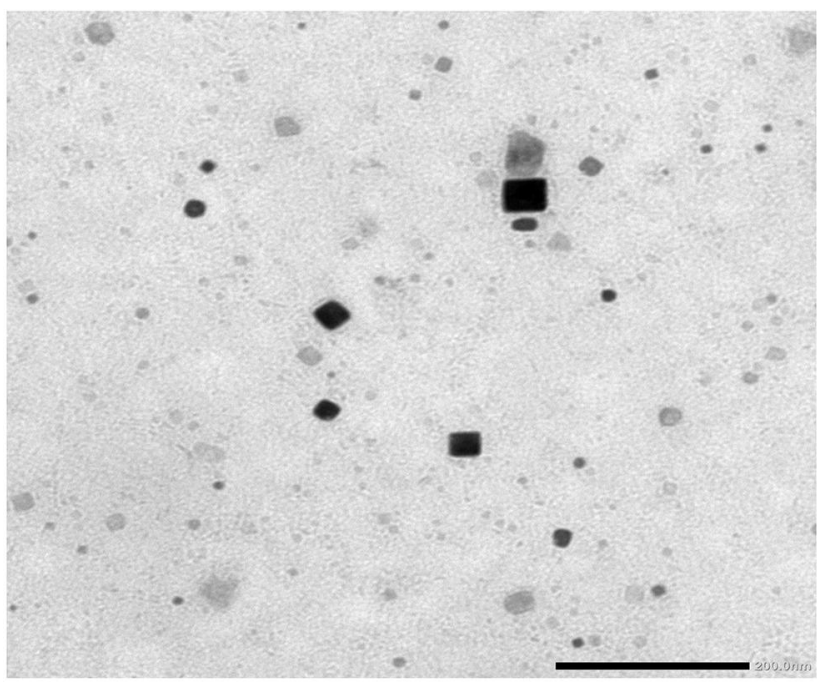

Figure I Transmission electron microscope imaging of thymoquinone-loaded cubosomes. increasing formulation percentage $(\mathrm{v} / \mathrm{v})$ was investigated. As shown in Figure 2A, the erythrocytes in the presence of the lowest percentage of cubosomal formulation behaved similar to that of the negative control with a slight increase in hemolytic activity up to $10.2 \pm 0.90 \%$ for highest volume of formulation added (Figure 2B). While treatment of Triton X100 solution (2\%) resulted in complete hemolysis due to absence of precipitation as seen in Figure 2A as a positive control. Moreover, optical microscopic study revealed intactness of erythrocytes after incubation with TQ-loaded cubosomes; the erythrocytes were comparable to the negative control (Figure 2), while the positive control microscopic images presented an absence of erythrocytes, thus confirming its complete hemolysis.

\section{TQ-Loaded Cubosomal Nanoparticles are More Toxic to Breast Cancer Cell Lines Than Free TQ at Sublethal Doses to Non-Tumorigenic Breast Cells}

The anticancer activity of TQ-loaded cubosomal nanoparticles was not tested previously against breast cancer cell lines, thus the range of active concentrations was determined, and their anticancer effect was investigated through MTT assay. Moreover, the blank cubosomes cytotoxicity was assessed and a comparison between the anticancer activity of TQloaded cubosomes and drug free cubosomes was performed. To achieve these aims, non-tumorigenic MCF-10A breast cells, aggressive metastatic breast cancer MDA-MB-231 cell line, and invasive breast ductal carcinoma MCF-7 cell line were treated with either free TQ, blank cubosomes or TQ-loaded cubosomes for 24 hours. The results revealed that the blank cubosomes and free TQ did not exert any cytotoxic effects on MCF-10A, the non-tumorigenic breast cells, at concentrations ranging from 1 to $30 \mu \mathrm{M}$ (Figure 3A). Furthermore, TQ-loaded cubosomes below $30 \mu \mathrm{M}$ were relatively non-cytotoxic to MCF-10A cells as cell viability was maintained at $77 \pm 9.30 \%$. Treatment of MCF-7 and MDAMB-231 breast cancer cells with increasing concentrations of the different formulations showed a significant dosedependent inhibition of cell viability in response to TQ or TQ-loaded cubosomes and the cytotoxic effect of TQ-loaded cubosomes was more pronounced in comparison to free TQ (Figure 3). In MCF-7 cells, TQ-loaded cubosomes induced a reduction in cell viability by $60 \pm 0.80 \%$ at $27.6 \mu \mathrm{M}$. On the contrary, free unencapsulated TQ was able to inhibit cell viability by only $30 \%$ at the same concentration (Figure 3B). Concerning MDA-MB-231 cell line, the viability was 


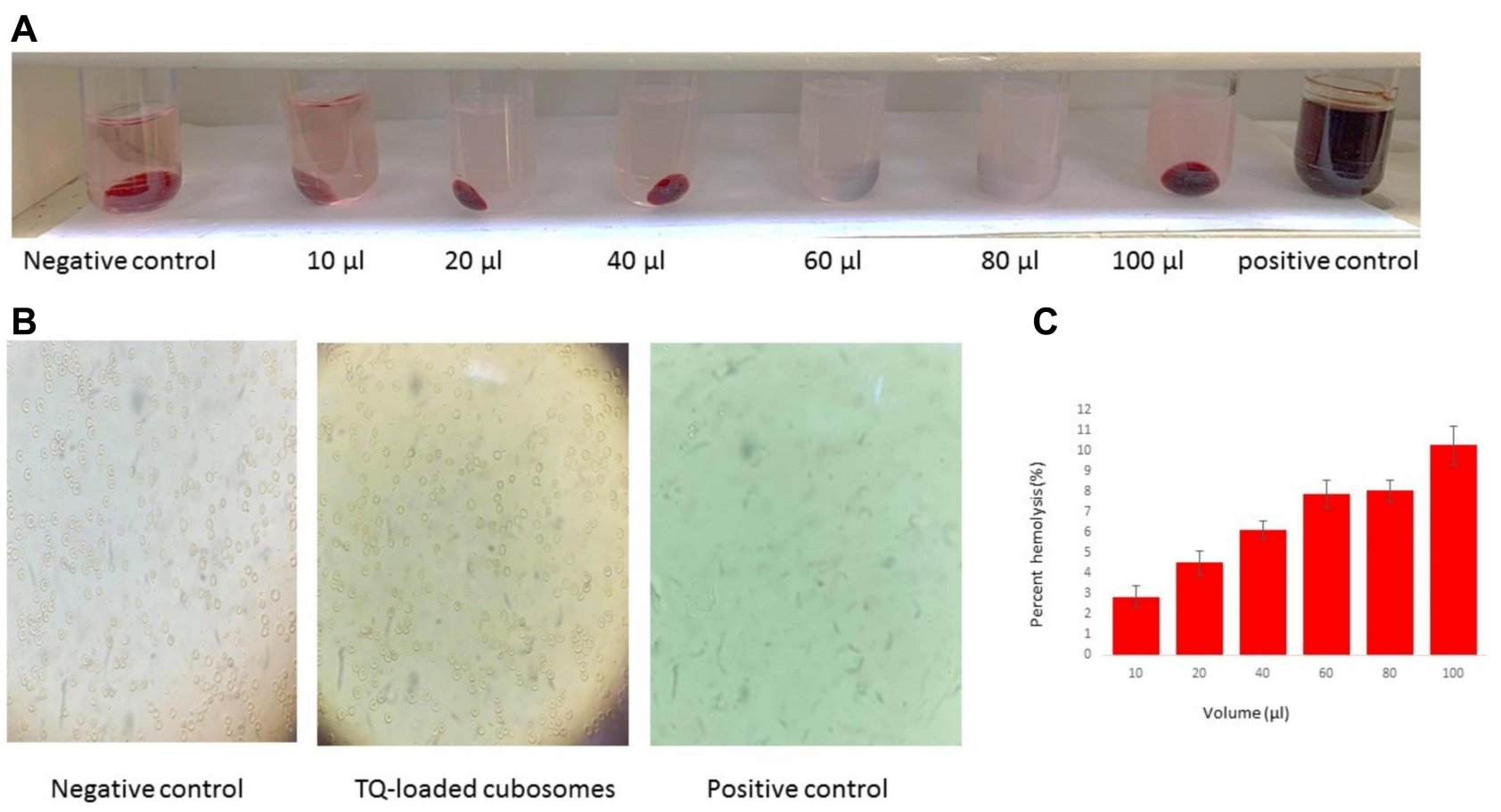

Figure 2 Hemolytic activity and hemolysis percentage of TQ-loaded cubosomes (A) photograph of erythrocytes treated with TQ-loaded cubosomes at different percentage (v/v), (B) photomicrograph of erythrocytes treated with PBS (negative control), TQ-loaded cubosomes, Triton X (positive control), (C) Data expressed as mean \pm SD of erythrocytes treated with different percentages of TQ-loaded cubosomes.

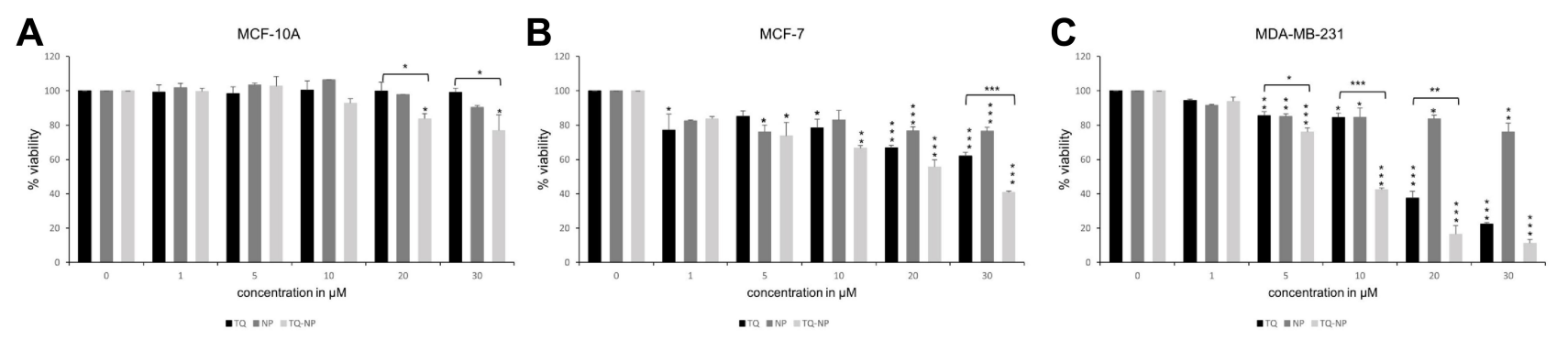

Figure 3 Toxicity of TQ-loaded cubosomes to non-tumorigenic breast cells and their anticancer effect in human breast cancer cells in comparison to free TQ. MTT assay showing the viability of (A) MCF-IOA normal breast cell line. (B) MCF-7 breast cancer cell line. (C) MDA-MB-23I aggressive breast cancer cell line. The cells were treated for $24 \mathrm{~h}$ with different concentrations of either TQ, or blank cubosomes or TQ-loaded cubosomes. Experiments were repeated three times, data are means $\pm \mathrm{SEM}$, asterisk indicates $\mathrm{p}<0.05$ with respect to the untreated control, bar and asterisk indicates $\mathrm{p}<0.05$ of TQ-loaded cubosomes with respect to TQ, ${ }^{*} \mathrm{p}<0.05$, $* * \mathrm{p}<0.0 \mathrm{I}, * * * \mathrm{p}<0.00 \mathrm{I}$.

observed to be decreased to $22 \pm 0.90 \%$ in the presence of free TQ in comparison to $11 \pm 2.30 \%$ with TQ-loaded cubosomes (Figure 3C). TQ and TQ-loaded cubosomes concentrations used in subsequent experiments represent the $\mathrm{IC}_{50}$ values obtained by the MTT assay (Table 2).

\section{Free TQ and TQ-Loaded Cubosomes Induce Apoptosis Through Caspase 3 Cleavage}

The cellular mechanism of the observed antitumor effects was investigated. The capability of TQ formulation to induce apoptosis was studied by DAPI staining and by quantifying the cleavage of caspase 3. The number of apoptotic bodies in both free TQ or TQ-loaded cubosomes treated cells increased as illustrated in Figure 4A. This enhanced apoptotic response was further confirmed by

Table 2 Calculated IC $C_{50}$ Values $(\mu \mathrm{m})$ from MTT Assay of Free TQ and TQ-Loaded Cubosomes in Both MCF-7 and MDA-MB-23I Cell Lines After Treatment with Concentrations Ranging from I to $30 \mu \mathrm{m}$

\begin{tabular}{|l|l|l|}
\hline \multirow{2}{*}{ Cell Line } & \multicolumn{2}{|l|}{ IC50 Values $(\boldsymbol{\mu M})$ MTT Assay $(\mathrm{I}-30 \mu \mathrm{M})$} \\
\cline { 2 - 3 } & TQ & TQ-Loaded Cubosomes \\
\hline MCF-7 & 55.20 & 27.60 \\
MDA-MB-23I & 27.60 & 7.80 \\
\hline
\end{tabular}




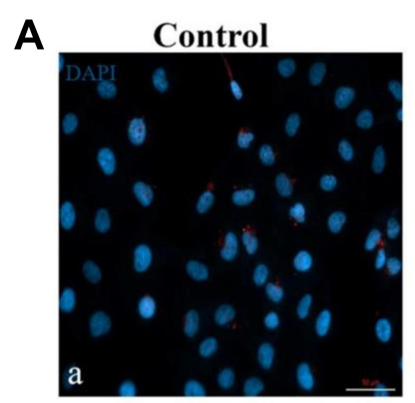

Cubosomes

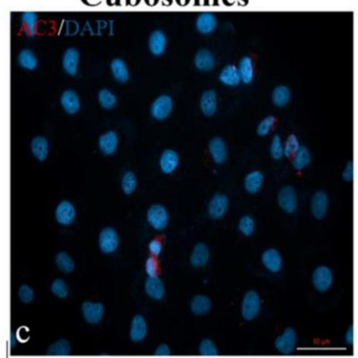

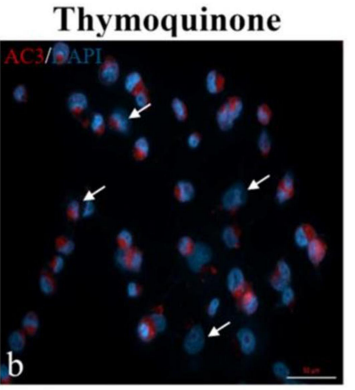

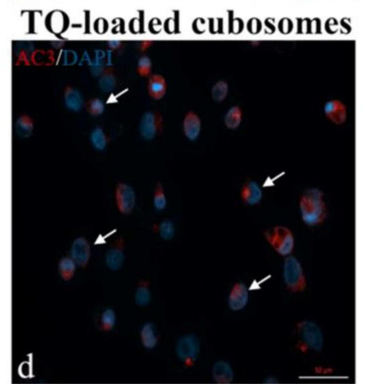

B

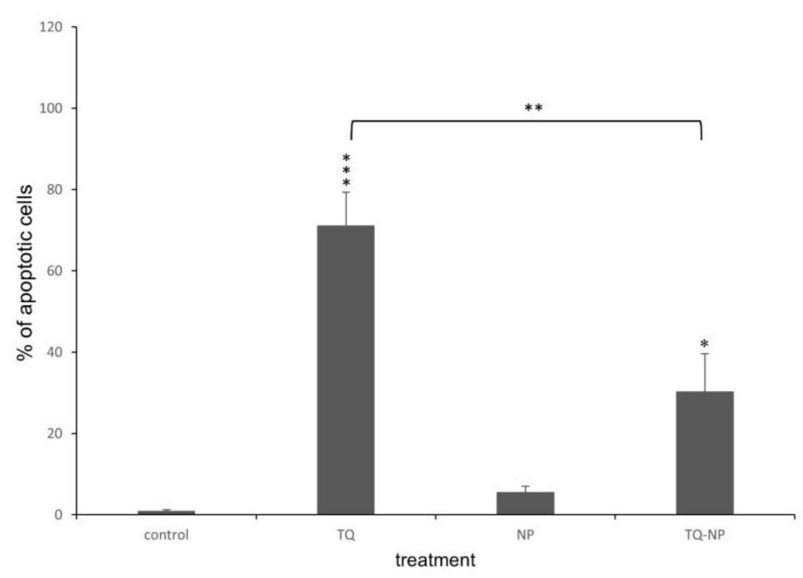

Figure 4 (A) Immunofluorescent analysis of active caspase 3 (AC3) expression in MDA-MB-23I cell line after $24 \mathrm{~h}$ of treatment with TQ and TQ-loaded cubosomes using the $I_{50}$ values obtained from MTT. Visualized by microscope Zeiss Axio, 40X oil immersion. (B) Active caspase 3 quantification in MDA-MB-23I cell line. Experiments were repeated three times, data are means \pm SEM, asterisk indicates $\mathrm{p}<0.05$ with respect to the untreated control, bar and asterisk indicates $\mathrm{p}<0.05$ of TQ-loaded cubosomes with respect to TQ, ${ }^{*}<0.05,{ }^{* *} p<0.01,{ }^{* *} p<0.001$. Visualized by microscope Zeiss Axio, $40 \mathrm{X}$ oil immersion. Arrows indicate apoptotic bodies in the nuclei stained by DAPI.

the significant increase in active caspase 3 in MDA-MB -231 cells treated with free TQ and TQ-loaded cubosomes. The percentage of cleaved caspase 3 was $71 \pm 8.30 \%$ and $30 \pm 9.40 \%$ in cells treated with free TQ and TQ-loaded cubosomes, respectively (Figure 4B), while the unloaded cubosomes resulted in a statistically non-significant change in the expression of active caspase 3 .

\section{TQ-Loaded Cubosomes are Internalized in Breast Cancer Cell Lines by Endocytosis}

Nile red was coupled to cubosomes in both their blank and drug encapsulated state to track their uptake and localization in breast cancer cell lines by fluorescent microscopy. After treatment of MCF-7 and MDA-MB-231 cell lines for 30 minutes, the microscopic images revealed that the cubosomal nanoparticles were taken up into the cytoplasm of both breast cancer cell lines (Figure 5A). In MCF-7 cells, blank cubosomes were evenly distributed around the nuclei, while TQ-loaded cubosomes had a punctate distribution as clearly visualized in Figure $5 \mathrm{~A}$.

The intracellular localization of TQ-loaded cubosomes was thoroughly investigated through labeling the nuclei with DAPI and staining the cells with caveolin (endosomal marker), LAMP (lysosomal marker), transferrin (marker of clathrin-mediated endocytosis) and EEA (marker of early endosomes) compared to the control untreated stained breast cancer cell lines (Supplementary Figure 1) to further determine the cellular trafficking mechanisms. Microscopical images revealed that cubosomes, in both their unloaded and TQ-loaded state, colocalized mainly with caveolin and transferrin (Figure 5B and Supplementary Figure 2), suggesting that the cubosomal nanoparticles access the breast cancer cell lines via caveolin and clathrin-dependent endocytosis.

\section{Storage Stability Evaluation}

The storage stability of TQ-loaded LCNPs were evaluated through monitoring of particle size, zeta potential and PDI. Over the studied three months period, the formulation was able to maintain its particle size with insignificant change $(104 \pm 8.52 \mathrm{~nm}$ at day 90$)$. The zeta potential as well as the PDI were consistent with the fresh formulation measurements.

\section{Discussion}

There are limited studies on the anticancer efficacy of TQloaded cubosomal nanoparticles and this is the first comprehensive study documenting their activity and uptake mechanisms. The overall aim of the project was to test the anticancer potential of novel cubosomal nanoparticles 

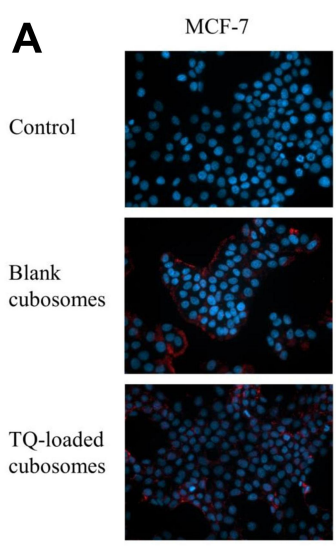

MDA-MB-231
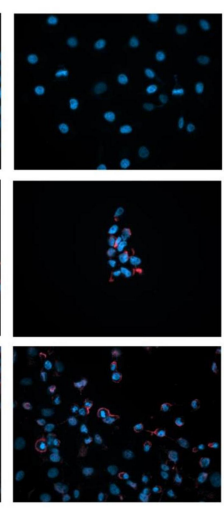

B

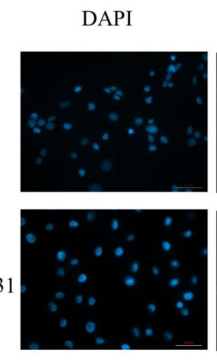

DAPI
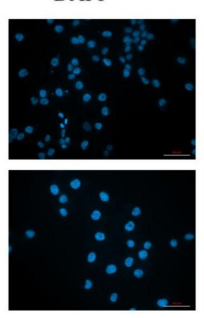

Nile Red
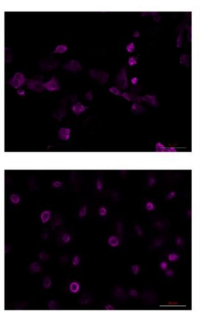

Nile Red
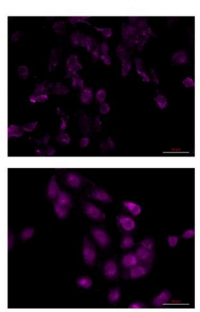

LAMP
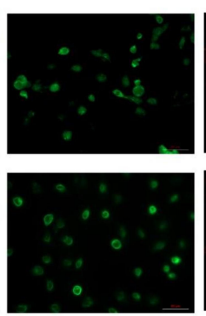

EEA-1
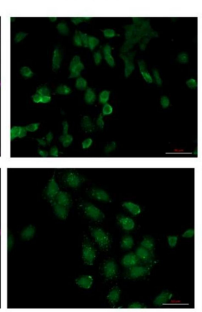

Caveolin

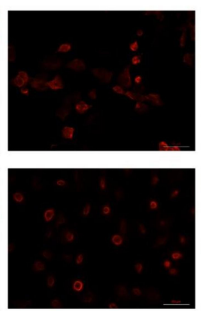

Transferrin
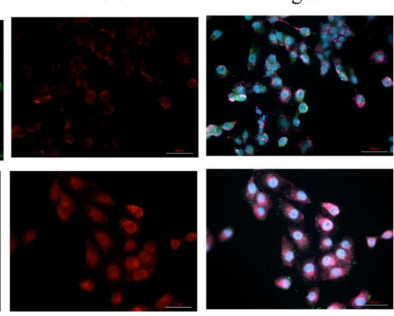

Merged
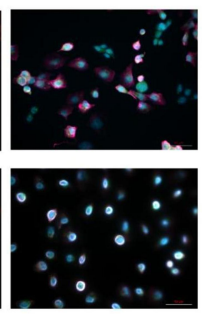

Merged

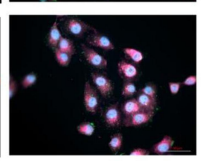

Figure 5 Mechanism of cellular uptake and trafficking of TQ-loaded cubosomes in human breast cancer cell lines. (A) Cellular uptake of the TQ and blank formulations in MCF-7 and MDA-MB-23I cell lines after 30 mins of treatment with the IC 50 concentrations. (B) Subcellular localization of TQ-loaded cubosomes in MCF-7 and MDA-MB $-23 \mathrm{I}$ cell lines. Cells were treated with $27.60 \mu \mathrm{M}$ (MCF-7) and $7.60 \mu \mathrm{M}$ (MDA-MB-23I) of TQ-loaded cubosomes for 30 mins. The cubosomal formulations were labeled with nile red for tracing inside the cells. Slides were labeled with DAPI, LAMP, Caveolin, EEA-I and Transferrin. Visualized by microscope Zeiss Axio, 40X oil immersion. Scale is $50 \mu \mathrm{m}$.

encapsulating TQ, an anticancer compound extracted from Nigella sativa, and compare their activity with free TQ in human breast cancer cell lines in vitro.

In the current study, thymoquinone self-assembled liquid crystalline nanoparticles were formulated through utilization of glyceryl monooleate as a cubic phase precursor lipid with PLX as a polymeric stabilizer. The lipid phase and stabilizer were mixed with the aqueous phase through emulsification method followed by high speed homogenization.

The particle size is a determinant factor for the cellular uptake of drug-loaded nanoparticles, thus exerting an important influence on the biopharmaceutical performance of the formulated cubosomes. Moreover, the values of the PDI reflect the degree of system homogeneity, where values closer to zero indicate a more uniform dispersion. $^{23}$ The formulated thymoquinone loaded cubosomes particle size was of nanometric size similar to those reported in literature. ${ }^{32}$ In Tian et al study, etoposide loaded-cubosomes had particle sizes of $173.6 \pm 1.60 \mathrm{~nm}$ with 0.174 PDI value; the consistent low PDI in various studies demonstrated the ability of cubosomes dispersion to maintain homogeneity, yet the observed particle size difference is clarified by the modified formulation and process parameters. This is further evident by Swarnakar et al study which highlighted the effect of the formulation factors as well as the process parameters as the surfactant nature and its concentration, drug loading as well as the sonication time on the cubosomes particle size and PDI. ${ }^{35}$

The zeta potential of the nanovesicles system reveals information about the stability of the vesicular system as well as its surface properties. High zeta potential values are preferred as they denote strong repellent interaction between the cubic nanoparticles, thus providing high physical stability as the repulsion forces prevent their coalescence and maintain a stable colloidal nanodispersion. The zeta potential of the various cubosomal nanoparticles were similar to those prepared in literature indicating good stability of the system. ${ }^{36}$ Furthermore, the observed high entrapment efficiency of TQ into the cubosomal sponge is a strategic aim in formulating this novel therapeutic system. This major asset holds a critical effect on the therapeutic efficacy of the final formulation. In this study, thymoquinone was entrapped within the cubic phase with high entrapment efficiency of $96.60 \pm 3.58 \% \mathrm{w} / \mathrm{w}$. This can be attributed to the high solubility of the hydrophobic drug within the lipid bilayers. This finding is in accordance with Nazaruk et al who reported a high entrapment efficiency $(92 \pm 40 \%)$ of doxorubicin in cubosomal formulation which was also attributed to the lipophilic nature of the system. ${ }^{23}$

To the best of the authors knowledge, this is the first report to assess the anticancer activity of a novel TQ-based cubosomal nanoparticles and compare its activity to the 
free drug in human breast cancer cell lines. The obtained results highlighted that the free TQ, blank cubosomes, and TQ-loaded cubosomes exerted non-toxic effects on the non-tumorigenic MCF-10A breast cells at doses up to 30 $\mu \mathrm{M}$. Interestingly, TQ-loaded cubosomes decreased the viability of human breast cancer MCF-7 and MDA-MB -231 cell lines more significantly than that induced by free TQ. Fluorescent microscopical images showed that the nanosystems were taken up by the cells and localized in their cytoplasmic compartment. The cubosomal nanoparticles co-localized with the endocytosis markers clathrin and caveolin, suggesting that endocytosis is the main mechanism of cellular trafficking.

The hemocompatibility testing of any parenteral formulation is mandatory, hence the hemolytic activity of the cubosomal formulation was assessed. The formulation compromised of GMO/PLX/TQ revealed a slight hemolytic activity of $3 \%$ at low concentrations, the safety profile was further depicted via detection of intact erythrocytes by light microscopic imaging, which indicated an acceptable safety profile of the formulation. These results are in accordance with that of Bode et al who studied the hemolytic activity of different cubic phase crystalline structure preparations and evaluated the excipients utilized in their formulations and found that the interaction of the dispersed phases with the blood compartment disclosed low but slight tendency toward hemolysis of cubosomes prepared with GMO/PLX407. ${ }^{37}$

There are limited studies on the anticancer efficacy of TQ-loaded cubosomal nanoparticles and this is the foremost comprehensive study documenting their activity and delineating their cellular uptake mechanisms. The cytotoxic activity of TQ on breast cancer cells is well documented. ${ }^{9}$ Several nanovehicles of TQ have been studied including liposomes, solid lipid nanoparticles, polymeric nanoparticles, and PEGylated nanoparticles, all of which showed augmented anticancer activity compared to the unencapsulated drug. ${ }^{38-42}$ The authors previously formulated and examined a polymeric TQ-loaded nanoparticles based on $\mathrm{PS}_{1600} \mathrm{PEO}_{1800}$ amphiphilic diblock polymers which exhibited suitable physicochemical characteristics for drug delivery including small particle size, high encapsulation efficiency, high loading capacity and stability. The polymeric nanoparticles formulation showed an enhanced anticancer activity, in comparison to free TQ, in MCF-7 and MDA-MB-231 breast cancer cell lines and was non-toxic to MCF-10A breast cell line; ${ }^{33}$ however, in vivo examination of such nanoformulations was hindered by the use of tetrahydrofuran which is a toxic vehicle. On the contrary, the current TQ-loaded cubosomal preparation is a water-based system which is safe for injection into animals and appears to be an appropriate drug delivery system as evidenced by its physicochemical characteristics. The size of the cubosomal nanoformulations was less than $100 \mathrm{~nm}$, which is the size recommended by the National Cancer Institute for cancer cell targeting. This size range is a prerequisite for successful delivery of the drug into the neoplastic cells while nanostructures with diameters less than $10 \mathrm{~nm}$ are susceptible to renal clearance and tissue extravasation, and nanoparticles with diameters greater than $100 \mathrm{~nm}$ are prone to opsonization by macrophages. ${ }^{16}$ In addition, the cubosomal formulation exhibited high encapsulation efficiency which indicates that a high percentage of TQ is being encapsulated into the liquid crystalline nanoarchitecture. In the developed system, the obtained $\mathrm{IC}_{50}$ values by MTT assay of TQ-loaded cubosomes were 2.0-3.5fold lower than that of free TQ in MCF-7 and MDA-MB-231 cell lines, respectively (Table 2). The observed ameliorated anticancer activity of TQ upon its encapsulation into the cubosomal nanoparticles indicates that the lipid nanostructures improved the drug delivery into the cells, and thus equivalent TQ anticancer activity could be obtained at lesser concentrations. This can be explained by the increased number of TQ molecules that are being delivered to the target site when present in cubosomes. Collectively, TQ-loaded cubosomal nanoparticles appear to form a better delivery platform than free TQ in both breast cancer cell lines.

In order to explore the cell death mechanism induced by TQ-loaded cubosomes, several approaches which included DAPI staining of nuclei coupled with microscopical examination aimed at detection of the extent of cell apoptosis and quantification of active caspase 3 using immunofluorescence techniques. Both TQ and TQ-loaded cubosomes were able to induce apoptosis in MDA-MB-231 as evidenced by the increased number of apoptotic bodies (DAPI staining) and the significant increase in cleaved caspase 3, while blank cubosomes had non-significant effects. Our results are in accordance with a Barkat et al study that had documented apoptosis induction by TQ in the triple-negative breast cancer (TNBC) with mutant p53, including MDA-MB-231 cell line. ${ }^{43}$ Treatment of MDAMB-231 cells with TQ caused cell cycle arrest at G1 phase and induced caspase-dependent and independent apoptosis through mitochondrial membrane permeabilization. ${ }^{44}$ 
Although, TQ-loaded cubosomes had a greater inhibitory effect on cell viability, free TQ caused a greater caspase 3 cleavage (apoptosis). This discrepancy could be due to the differences in the cellular effects of TQ vs TQ-loaded cubosomes on breast cancer cells where the latter may be able to induce cell cycle arrest more than apoptosis, while the former may have a pronounced apoptotic effect in comparison to its effects on cell cycle arrest, a hypothesis that should be investigated and confirmed with further experimental research.

TQ is known to modulate various signaling pathways and essential oncogenic molecules which play a role in cancer initiation, invasion, angiogenesis and metastasis. In a study focusing on myeloid leukemia and pancreatic ductal adenocarcinoma, TQ inhibited TNF $\alpha$ induced NF-KB activation thus suppressing transcription of proinflammatory gene products such as $\mathrm{COX}-2$, monocyte chemoattractant protein (MCP-1), TNF- $\alpha$ and IL-1ß. TQ inhibited IKK activation, prevented IKB degradation, in addition to inhibiting the nuclear translocation of $\mathrm{p} 65$ and binding to DNA. ${ }^{45,46}$ In a study investigating TQ anticancer activity in myelogenous leukemia, TQ inhibited NF$\mathrm{KB}$ and decreased matrix metalloproteinase (MMP)-9 levels. $^{47}$ Another study reported inhibition of NF-KB phosphorylation and the controlled gene products by TQ in human hepatocellular carcinoma. ${ }^{48}$ In Hodgkin's lymphoma L428 cell lines, TQ produced anti NF-KB activity, ${ }^{49}$ and in colon cancer it decreased phospho-p65 levels and the expression of Bcl-2, c-Myc and vascular endothelial growth factor. ${ }^{50}$

Knowing that the STATs family play a role in cytokine signaling which are involved in cell growth regulation, differentiation and angiogenesis, studies have reported the ability of TQ to regulate this cascade system. Li et al demonstrated the ability of TQ to inhibit constitutive and IL-6 inducible STAT3 phosphorylation. It was also found that TQ induced the expression of Srchomolgy-2 phosphate $2 .{ }^{51}$ In gastric cell lines, TQ inhibited the phosphorylation of STAT3, this down regulation was associated with phospho-JAK2 and Src suppression. ${ }^{52}$ In breast cancer cell lines, TQ induced cell cycle arrest and apoptosis as well as inhibited the expression of CXCR4 in MDA-MB $-231 .^{53,54}$

In ovarian cancer cells, TQ inhibited the growth of Caov-3, induced ROS production and blocked MMP leading to apoptosis mediated cell death. ${ }^{55}$ In human medulloblastoma cell lines, TQ suppressed cell proliferation, and induced ROS generation with induction of caspase-3 mediated apoptosis. ${ }^{56}$ In renal cell carcinoma Caki cells, TQ induced apoptosis through the down regulation of Bcl2 and c-FLIP, increased ROS production and decreased MMP expression. ${ }^{57}$ Moreover TQ was shown to suppress human Lovo colon cancer cell proliferation through down regulating the $p$-PI3 $\mathrm{K}, p$-GSK3ß, $p$-Akt and $\beta$-catenin expression, thus inhibiting downstream COX-2 expression. $^{58}$

The observation of cubosomal nanoparticles internalization by the breast cancer cells and localization in the cytoplasm suggested that the nanoformulation has the capacity to reach the cellular target which further explains their reported enhanced anticancer activity. The uptake of the cubosomal nanoparticles into the cells is indeed essential for loaded drug delivery and therapeutic efficacy. Predominantly, thymoquinone encapsulation into fluorescent cubosomal nanoparticles (Nile red-labeled) enabled their visualization inside the cells which provided an additional benefit over the free form of the drug. As such, thorough information about the tumor status, treatment regimens and tumor response to therapy monitoring could be obtained, ${ }^{59}$ which in turn enhances the possibility of TQ translation to the clinic.

Interestingly, in vitro uptake fluorescent microscopical examination illustrated an obvious difference in the distribution pattern of the formulations whereby blank cubosomal nanoparticles were evenly distributed while TQ-encapsulated cubosomal nanoparticles showed a punctate distribution. Various factors can affect the nanoparticlescellular uptake, trafficking and distribution. Among them are their physicochemical characteristics, including their sizes, charge, surface chemistry, and ionic strength, in addition to biological and experimental factors. ${ }^{60}$ Minor alterations of the nanoparticles size, solubility and composition specifically hydrophilicity/hydrophobicity may contribute significantly to their uptake. $^{60,61}$ Add to this, TQ is a highly hydrophobic compound and its bedding into cubosomes may induce modification of its internal structure in addition to minor changes in its size especially when considering that the nanoparticles first interact with the surrounding microenvironment. The microenvironmental properties, such as the secreted factors by cells and $\mathrm{pH}$ level can affect the nanoparticles characteristics, its interaction with cells, and subsequent uptake and trafficking within the cells. ${ }^{60}$ The mentioned facts could clarify the observed difference in the distribution pattern of the blank cubosomes vs TQloaded cubosomes. 
Endocytosis appeared to be the mechanism by which TQ-loaded cubosomes were uptaken by the cells since the formulations co-localized with the antibodies targeting caveolae, clathrin coated pits, early endosomes and lysosomes. Fakhoury et al mechanistic studies proved that polymer TQ-loaded nanoparticles were taken up into the cells by clathrin and caveolin-mediated endocytosis. ${ }^{33}$ The route and mechanism of entry of the nanoparticles determine their fate inside the cells. Nanoparticles taken up by endocytosis are first carried by early endosomes that eventually mature into late endosomes, which then fuse with lysosomes forming the endo-lysosomal vesicles that contain hydrolytic enzymes. ${ }^{62}$ Most often, nanoparticles taken up by clathrin mediated endocytosis end up in the endolysosomal pathway leading to the degradation of the cargo. ${ }^{60,62}$ However, nanoparticles taken up by caveosomes may escape lysosomal degradation and be released into the cytoplasm which leads to enhanced drug delivery and therapeutic potential. ${ }^{60,62}$ This may represent another possible rationale for the observed improved anticancer activity of TQ-loaded cubosomes compared to free thymoquinone.

The storage stability of the TQ-loaded cubosomes was evaluated through monitoring the particle size, zeta potential and PDI. Over the studied three months period, the formulation maintained its particle size with insignificant change (104 $\pm 8.52 \mathrm{~nm}$ at day 90$)$. The zeta potential as well as the PDI were consistent with the fresh sample measurements. The results assured the stability of the prepared TQ-loaded cubosomal nanoparticles under storage conditions. The obtained results were in accordance with an Elgindy et al study where the optimized cubosomal formulation maintained its stability for three months. ${ }^{29}$

In summary, thymoquinone anticancer activity against breast cancer cells was enhanced upon its encapsulation into self-assembled cubic liquid crystalline nanoparticles. Several limitations facing thymoquinone use, among which its low bioavailability and binding to plasma proteins, were overcome via its encapsulation into the liquid nanoparticles. Besides, the visualization and tracking of the drug in formulation became possible unlike the free thymoquinone. Our results suggest a novel platform for encapsulating thymoquinone into cubosomal nanoparticles would promote its future translation to the clinic. The signaling pathways and the key targeted molecules that are responsible for the enhanced activity of the cubosomal nanoparticles against breast cancer cell lines will be investigated.

\section{Acknowledgments}

This research has been funded with support from the National Council for Scientific Research in Lebanon (CNRS-L) and the American University of Beirut (AUB) and by the Swedish Research Council (Swedish Research Links 102861).

The authors would like to acknowledge the National Council for Scientific Research of Lebanon (CNRS-L) and Beirut Arab University (BAU) for granting a joint doctoral fellowship (CNRS-L/BAU) to Jana K. Alwattar.

The authors are grateful to the members of the Central Research Science Laboratory and the Medical School Core Laboratory at the American University of Beirut for their technical assistance.

\section{Disclosure}

Rana Sarieddine reports grants from National Council for Scientific Research in Lebanon (CNRS-L) and Swedish Research Council (Swedish Research Links 102861), and non-financial support from American University of Beirut (AUB), during the conduct of the study. The authors report no other potential conflicts of interest in this work.

\section{References}

1. Dagogo-Jack I, Shaw AT. Tumour heterogeneity and resistance to cancer therapies. Nat Rev Clin Oncol. 2018;15(2):81-94. doi:10.10 38/nrclinonc. 2017.166

2. Siegel RL, Miller KD, Jemal A. Cancer statistics, 2019. CA Cancer J Clin. 2019;69(1):7-34. doi:10.3322/caac.21551

3. Bodai BI, Tuso P. Breast cancer survivorship: a comprehensive review of long-term medical issues and lifestyle recommendations. Perm J. 2015;19(2):48-79. doi:10.7812/TPP/14-241

4. Zhou HB, Yan Y, Sun YN, Zhu JR. Resveratrol induces apoptosis in human esophageal carcinoma cells. World J Gastroenterol. 2003;9 (3):408-411. doi:10.3748/wjg.v9.i3.408

5. Boncel S, Herman AP, Budniok S, et al. In vitro targeting and selective killing of $\mathrm{t} 47 \mathrm{~d}$ breast cancer cells by purpurin and 5-fluorouracil anchored to magnetic cnts: nitrene-based functionalization versus uptake, cytotoxicity, and intracellular fate. ACS Biomater Sci Eng. 2016;2:1273-1285. doi:10.1021/acsbiomaterials.6b00197

6. Swaminathan S, Pastero L, Serpe L, et al. Cyclodextrin-based nanosponges encapsulating camptothecin: physicochemical characterization, stability and cytotoxicity. Eur J Pharm Biopharm. 2010;74 (2):193-201. doi:10.1016/j.ejpb.2009.11.003

7. Pushpalatha R, Selvamuthukumar S, Kilimozhi D. Cross-linked, cyclodextrin-based nanosponges for curcumin delivery Physicochemical characterization, drug release, stability and cytotoxicity. J Drug Deliv Sci Technol. 2018;45:45-53. doi:10.1016/ j.jddst.2018.03.004

8. Liebmann JE, Cook JA, Lipschultz C, Teague D, Fisher J, Mitchell JB. Cytotoxic studies of pacfitaxel $\left(\right.$ Taxol $\left.^{\circledR}\right)$ in human tumour cell lines. $\mathrm{Br}$ J Cancer. 1993;68(6):1104-1109. doi:10.1038/bjc.1993.488

9. Schneider-Stock R, Fakhoury IH, Zaki AM, El-Baba CO, GaliMuhtasib HU. Thymoquinone: fifty years of success in the battle against cancer models. Drug Discov Today. 2014;19(1):18-30. doi:10.1016/j.drudis.2013.08.021 
10. Yimer EM, Tuem KB, Karim A, Ur-Rehman N, Anwar F. Nigella sativa L. (Black Cumin): a promising natural remedy for wide range of illnesses. Evid Based Complement Alternat Med. 2019;6:1-16. doi:10.1155/2019/1528635

11. Jain RA. The manufacturing techniques of various drug loaded biodegradable poly(lactide-co-glycolide) (PLGA) devices. Biomaterials 2000;21(23):2475-2490. doi:10.1016/S0142-9612(00)00115-0

12. El-Najjar N, Ketola RA, Nissilä T, et al. Impact of protein binding on the analytical detectability and anticancer activity of Thymoquinone. J Chem Biol. 2011;4(3):97-107. doi:10.1007/ s12154-010-0052-4

13. Kumari A, Yadav SK, Yadav SC. Biodegradable polymeric nanoparticles based drug delivery systems. Colloids Surfaces B Biointerfaces. 2010;75(1):1-18. doi:10.1016/j.colsurfb.2009.09.001

14. Wang AZ, Langer R, Farokhzad OC. Nanoparticle delivery of cancer drugs. Annu Rev Med. 2012;63:185-198. doi:10.1146/annurev-med $-040210-162544$

15. Petschauer JS, Madden AJ, Kirschbrown WP, Song G, Zamboni WC. The effects of nanoparticle drug loading on the pharmacokinetics of anticancer agents. Nanomedicine. 2015;10(3):447-463. doi:10.2217/ nnm. 14.179

16. D'Souza SA. Review of in vitro drug release test methods for nano-sized dosage forms. Adv Pharm. 2014;2014:1-12. doi:10.11 $55 / 2014 / 304757$

17. Siemann DW. The unique characteristics of tumor vasculature and preclinical evidence for its selective disruption by tumor-vascular disrupting agents. Cancer Treat Rev. 2011;37(1):63-74. doi:10.101 6/j.ctrv.2010.05.001

18. Viallard C, Larrivée B. Tumor angiogenesis and vascular normalization: alternative therapeutic targets. Angiogenesis. 2017;20: 409-426. doi:10.1007/s10456-017-9562-9

19. Park JW. Liposome-based drug delivery in breast cancer treatment. Breast Cancer Res. 2002;4(3):95-99. doi:10.1186/bcr432

20. Dora CP, Trotta F, Kushwah V, et al. Potential of erlotinib cyclodextrin nanosponge complex to enhance solubility, dissolution rate, in vitro cytotoxicity and oral bioavailability. Carbohydr Polym. 2016;137:339-349. doi:10.1016/j.carbpol.2015.10.080

21. Fan T, Li M, Wu X, Li M, Wu Y. Preparation of thermoresponsive and $\mathrm{pH}$-sensitivity polymer magnetic hydrogel nanospheres as anticancer drug carriers. Colloids Surfaces B Biointerfaces. 2011;88 (2):593-600. doi:10.1016/j.colsurfb.2011.07.048

22. Oerlemans C, Bult W, Bos M, Storm G, Nijsen JFW, Hennink WE. Polymeric micelles in anticancer therapy: targeting, imaging and triggered release. Pharm Res. 2010;27(12):2569-2589. doi:10.1007/ s11095-010-0233-4

23. Nazaruk E, Majkowska-Pilip A, Bilewicz R. Lipidic cubic-phase nanoparticles - cubosomes for efficient drug delivery to cancer cells. Chempluschem. 2017;82(4):570-575. doi:10.1002/cplu.20160 0534

24. Murgia S, Bonacchi S, Falchi AM, et al. Drug-loaded fluorescent cubosomes: versatile nanoparticles for potential theranostic applications. Langmuir. 2013;29(22):6673-6679. doi:10.1021/la40 $1047 \mathrm{a}$

25. Mohyeldin SM, Mehanna MM, Elgindy NA. Superiority of liquid crystalline cubic nanocarriers as hormonal transdermal vehicle: comparative human skin permeation-supported evidence. Expert Opin Drug Deliv. 2016;13(8):1049-1064. doi:10.1080/17425247.2016.11 82490

26. Garg G, Saraf S, Saraf S. Cubosomes: an overview. Biol Pharm Bull. 2007;30(2):350-353. doi:10.1248/bpb.30.350

27. Pan X, Han K, Peng X, et al. Nanostructed cubosomes as advanced drug delivery system. Curr Pharm Des. 2013;19(35):6290-6297. doi:10.2174/1381612811319350006

28. Kluzek M, Tyler AII, Wang S, et al. Influence of a $\mathrm{pH}$-sensitive polymer on the structure of monoolein cubosomes. Soft Matter. 2017;13:7571-7577. doi:10.1039/c7sm01620d
29. Elgindy NA, Mehanna MM, Mohyeldin SM. Self-assembled nano-architecture liquid crystalline particles as a promising carrier for progesterone transdermal delivery. Int J Pharm. 2016;501(1-2):167-179. doi:10.1016/j.ijpharm.2016.01.049

30. Deshpande S, Singh N. Influence of cubosome surface architecture on its cellular uptake mechanism. Langmuir. 2017;33(14):35 09-3516. doi:10.1021/acs.langmuir.6b04423

31. Han S, Shen JQ, Gan Y, et al. Novel vehicle based on cubosomes for ophthalmic delivery of flurbiprofen with low irritancy and high bioavailability. Acta Pharmacol Sin. 2010;31(8):990-998. doi:10.10 38/aps. 2010.98

32. Tian Y, Li JC, Zhu JX, et al. Folic acid-targeted etoposide cubosomes for theranostic application of cancer cell imaging and therapy. Med Sci Monit. 2017;23:2426-2435. doi:10.12659/MSM.904683

33. Fakhoury I, Saad W, Bouhadir K, Nygren P, Schneider-Stock R, GaliMuhtasib H. Uptake, delivery, and anticancer activity of thymoquinone nanoparticles in breast cancer cells. $J$ Nanoparticle Res. 2016;18(7):1-16. doi:10.1007/s11051-016-3517-8

34. Barauskas J, Cervin C, Jankunec M, et al. Interactions of lipid-based liquid crystalline nanoparticles with model and cell membranes. Int J Pharm. 2010;391(1-2):284-291. doi:10.1016/j.ijpharm.2010.03. 016

35. Swarnakar NK, Thanki K, Jain S. Bicontinuous cubic liquid crystalline nanoparticles for oral delivery of doxorubicin: implications on bioavailability, therapeutic efficacy, and cardiotoxicity. Pharm Res. 2014;31(5):1219-1238. doi:10.1007/s11095-013-1244-8

36. Caltagirone C, Falchi AM, Lampis S, et al. Cancer-cell-targeted theranostic cubosomes. Langmuir. 2014;30(21):6228-6236. doi:10.10 21/la501332u

37. Bode JC, Kuntsche J, Funari SS, Bunjes H. Interaction of dispersed cubic phases with blood components. Int J Pharm. 2013;448 (1):87-95. doi:10.1016/j.ijpharm.2013.03.016

38. Pathan SA, Jain GK, Zaidi SMA, et al. Stability-indicating ultra-performance liquid chromatography method for the estimation of thymoquinone and its application in biopharmaceutical studies. Biomed Chromatogr. 2011;25(5):613-620. doi:10.1002/ bmc. 1492

39. Odeh F, Ismail SI, Abu-Dahab R, Mahmoud IS, Al Bawab A. Thymoquinone in liposomes: a study of loading efficiency and biological activity towards breast cancer. Drug Deliv. 2012;19 (8):371-377. doi:10.3109/10717544.2012.727500

40. Bhattacharya S, Ahir M, Patra P, et al. PEGylated-thymoquinonenanoparticle mediated retardation of breast cancer cell migration by deregulation of cytoskeletal actin polymerization through miR-34a. Biomaterials. 2015;51:91-107. doi:10.1016/j.biomaterials.2015.01. 007

41. Ng WK, Saiful Yazan L, Yap LH, Wan nor Hafiza WAG, How CW, Abdullah R. Thymoquinone-loaded nanostructured lipid carrier exhibited cytotoxicity towards breast cancer cell lines (MDA-MB-231 and MCF-7) and cervical cancer cell lines (HeLa and SiHa). Biomed Res Int. 2015;2015:1-10. doi:10.1155/ 2015/263131

42. Ballout F, Habli Z, Rahal ON, Fatfat M, Gali-Muhtasib H. Thymoquinone-based nanotechnology for cancer therapy: promises and challenges. Drug Discov Today. 2018;23(5):1089-1098. doi:10. 1016/j.drudis.2018.01.043

43. Barkat MA, Harshita H, Ahmad J, Khan MA, Beg S, Ahmad,FJ. Insights into the targeting potential of thymoquinone for therapeutic intervention against triple-negative breast cancer. Curr Drug Targets. 2018;19(1):70-80. doi:10.2174/138945011866617061209 5959

44. Sutton KM, Greenshields AL, Hoskin DW. Thymoquinone, a bioactive component of black caraway seeds, causes G1 phase cell cycle arrest and apoptosis in triple-negative breast cancer cells with mutant p53. Nutr Cancer. 2014;66(3):408-418. doi:10.1080/ 01635581.2013 .878739 
45. Sethi G, Ahn KS, Aggarwal BB. Targeting nuclear factor- $\mathrm{kB}$ activation pathway by thymoquinone: role in suppression of antiapoptotic gene products and enhancement of apoptosis. Mol Cancer Res. 2008;6(6):1059-1070. doi:10.1158/1541-7786.MCR07-2088

46. Chehl N, Chipitsyna G, Gong Q, Yeo CJ, Arafat HA. Antiinflammatory effects of the Nigella sativa seed extract, thymoquinone, in pancreatic cancer cells. Hpb. 2009;11(5):373-381. doi:10.11 11/j.1477-2574.2009.00059.x

47. Ravindran J, Nair HB, Sung B, Prasad S, Tekmal RR, Aggarwal BB. RETRACTED: thymoquinone poly (lactide-co-glycolide) nanoparticles exhibit enhanced anti-proliferative, anti-inflammatory, and chemosensitization potential. Biochem Pharmacol. 2010;79(11):16 40-1647. doi:10.1016/j.bcp.2010.01.023

48. Ashour AE, Abd-Allah AR, Korashy HM, et al. Thymoquinone suppression of the human hepatocellular carcinoma cell growth involves inhibition of IL-8 expression, elevated levels of TRAIL receptors, oxidative stress and apoptosis. Mol Cell Biochem. 2014;389(1-2):85-98. doi:10.1007/s11010-013-1930-1

49. Agbaria R, Gabarin A, Dahan A, Ben-Shabat S. Anticancer activity of Nigella sativa (black seed) and its relationship with the thermal processing and quinone composition of the seed. Drug Des Devel Ther. 2015;9:3119-3124. doi:10.2147/DDDT. S82938

50. Zhang L, Bai Y, Yang Y. Thymoquinone chemosensitizes colon cancer cells through inhibition of NF-kB. Oncol Lett. 2016;12 (4):2840-2845. doi:10.3892/ol.2016.4971

51. Li F, Rajendran P, Sethi G. Thymoquinone inhibits proliferation, induces apoptosis and chemosensitizes human multiple myeloma cells through suppression of signal transducer and activator of transcription 3 activation pathway. Br $J$ Pharmacol. 2010;161 (3):541-554. doi:10.1111/j.1476-5381.2010.00874.x

52. Zhu WQ, Wang J, Guo XF, Liu Z, Dong WG. Thymoquinone inhibits proliferation in gastric cancer via the STAT3 pathway in vivo and in vitro. World J Gastroenterol. 2016;22(16):4149-4159. doi:10.3748/ wjg.v22.i16.4149
53. Shanmugam MK, Arfuso F, Kumar AP, et al. Modulation of diverse oncogenic transcription factors by thymoquinone, an essential oil compound isolated from the seeds of Nigella sativa Linn. Pharmacol Res. 2018;129:357-364. doi:10.1016/j.phrs.2017.11.023

54. Shanmugam MK, Ahn KS, Hsu A, et al. Thymoquinone inhibits bone metastasis of breast cancer cells through abrogation of the CXCR4 signaling axis. Front Pharmacol. 2018;9. doi:10.3389/fphar.2018.01294

55. Taha MME, Sheikh BY, Salim LZA, et al. Thymoquinone induces apoptosis and increase ROS in ovarian cancer cell line. Cell Mol Biol. 2016;62(6):97-101. doi:10.14715/cmb/2016.62.6.18

56. Ashour AE, Ahmed AF, Kumar A, et al. Thymoquinone inhibits growth of human medulloblastoma cells by inducing oxidative stress and caspase-dependent apoptosis while suppressing NF- $\mathrm{kB}$ signaling and IL-8 expression. Mol Cell Biochem. 2016;416(1-2):141-155. doi:10.1007/s11010-016-2703-4

57. Park EJ, Chauhan AK, Min KJ, Park DC, Kwon TK. Thymoquinone induces apoptosis through downregulation of c-FLIP and Bcl-2 in renal carcinoma caki cells. Oncol Rep. 2016;36(4):2261-2267. doi:10.3892/or.2016.5019

58. Hsu HH, Chen MC, Day CH, et al. Thymoquinone suppresses migration of LoVo human colon cancer cells by reducing prostaglandin E2 induced COX-2 activation. World $J$ Gastroenterol. 2017;23 (7):1171-1179. doi:10.3748/wjg.v23.i7.1171

59. Kievit FM, Zhang M. Cancer nanotheranostics: improving imaging and therapy by targeted delivery across biological barriers. Adv Mater. 2011;23(36):17-47. doi:10.1002/adma.201102313

60. Behzadi S, Serpooshan V, Tao W, et al. Cellular uptake of nanoparticles: journey inside the cell. Chem Soc Rev. 2017;46 (14):4218-4244. doi:10.1039/c6cs00636a

61. De Jong WH, Borm PJ. Drug delivery and nanoparticles:applications and hazards. Int J Nanomedicine. 2008;3(2):133-149. doi:10.2147/ IJN.S596

62. Foroozandeh P, Aziz AA. Insight into cellular uptake and intracellular trafficking of nanoparticles. Nanoscale Res Lett. 2018;13(1):1-12. doi:10.1186/s11671-018-2728-6
International Journal of Nanomedicine

\section{Publish your work in this journal}

The International Journal of Nanomedicine is an international, peerreviewed journal focusing on the application of nanotechnology in diagnostics, therapeutics, and drug delivery systems throughout the biomedical field. This journal is indexed on PubMed Central, MedLine, CAS, SciSearch ${ }^{\mathbb{B}}$, Current Contents ${ }^{\mathbb{B}} /$ Clinical Medicine, $^{2}$
Journal Citation Reports/Science Edition, EMBase, Scopus and the Elsevier Bibliographic databases. The manuscript management system is completely online and includes a very quick and fair peer-review system, which is all easy to use. Visit http://www.dovepress.com/ testimonials.php to read real quotes from published authors. 